\title{
MIND at Neutrino Factories
}

\author{
Ryan Bayes** \\ University of Glasgow, Glasgow, United Kingdom \\ E-mail: ryan.bayes@glasgow.ac.uk
}

\begin{abstract}
Magnetized iron calorimeters have been previously used in neutrino detection applications, with MINOS being a good example. This technology provides the benefits of excellent charge and particle identification while being trivial to scale up in mass. These properties make a magnetized iron neutrino detector (MIND) the ideal far detector for neutrino factory applications. A full simulation of MIND has been produced to evaluate its detector response. The digitized simulation is subject to a full reconstruction of muon tracks. A multivariate analysis was developed to select muon tracks with a high purity to reduce backgrounds from charge and flavour mis-identification. The detector response and background suppression is optimized for the requirements for the specific experiment and will be discussed. The sensitivity of oscillation physics experiments using a MIND at a neutrino factory will be discussed using the response derived from this analysis will be discussed with a focus on leptonic CP violation.
\end{abstract}

16th International Workshop on Neutrino Factories and Future Neutrino Beam Facilities 25 -30 August, 2014

University of Glasgow, United Kingdom

* Speaker.

${ }^{\dagger}$ A footnote may follow. 


\section{Introduction}

Magnetized iron neutrino detectors (MIND) have been used in a number of applications because of their high mass and high muon detection efficiency[1,2,3]. These properties coupled with their cost effectiveness and easy scalability make MIND particularly attractive for neutrino oscillation measurements at a neutrino factory as studied by the Neutrino Factory International Design Study (IDS-NF)[4]. The great benefit of neutrino factories in general is that they provides a pure beam composed of exactly two neutrino species in equal quantities from muon decays in flight with no source backgrounds. Because a neutrino factory can store muons of either charge sign, the potential exists to make measurements of twelve different oscillation channels, as listed in Table 1, which are separable by interaction flavour or charge. The most promising of these channels is the Golden channel $\left(\bar{v}_{e} \rightarrow \bar{v}_{\mu}\right.$, which produces a clean appearance signal characterized by a muon with a charge opposite to that of the muon beam source, called the wrong-sign muon signal. The primary limitation to a measurement based on such a signal is its ability to select events based on the charge of the lepton in the final state.

\begin{tabular}{|l|c|c|}
\hline \hline & Store $\mu^{+}$ & Store $\mu^{-}$ \\
\hline Golden Channel & $v_{e} \rightarrow v_{\mu}$ & $\bar{v}_{e} \rightarrow \bar{v}_{\mu}$ \\
$v_{e}$ Disappearance Channel & $v_{e} \rightarrow v_{e}$ & $\bar{v}_{e} \rightarrow \bar{v}_{e}$ \\
Silver Channel & $v_{e} \rightarrow v_{\tau}$ & $\bar{v}_{e} \rightarrow \bar{v}_{\tau}$ \\
\hline Platinum Channel & $\bar{v}_{\mu} \rightarrow \bar{v}_{e}$ & $v_{\mu} \rightarrow v_{e}$ \\
$v_{\mu}$ Disappearance Channel & $\bar{v}_{\mu} \rightarrow \bar{v}_{\mu}$ & $v_{\mu} \rightarrow v_{\mu}$ \\
Dominant Oscillation & $\bar{v}_{\mu} \rightarrow \bar{v}_{\tau}$ & $v_{\mu} \rightarrow v_{\tau}$ \\
\hline \hline
\end{tabular}

Table 1: Oscillation channels available to an experiment at a neutrino factory.

The measurement of $\mathrm{CP}$ violation in the lepton sector has now become the great challenge of neutrino oscillation physics following the recent measurement of $\theta_{13}[5]$. The neutrino factory design was re-optimized to use a stored muon energy of $10 \mathrm{GeV}$ and a baseline of $2000 \mathrm{~km}$. To evaluate the potential of a realistic experiment at this facility a simulation of a MIND has been developed by the IDS-NF collaboration[6]. Based on this analysis it is known that a neutrino factory provides the best discovery potential of the proposed facilities because of its small anticipated beam line systematics.

\section{Simulation and Reconstruction}

The MIND simulation software was built using a compartmentalized approach. The GENIE simulation (version 2.6.4)[7] was used to produce the neutrino events within the detector materials. These events were then placed within a detector geometry simulated with the GEANT4 (release 9.4.p01) package[8] using the QGSP_BERT physics lists for the propagation of final state particles. The MIND geometry was built up of modules composed of alternating sheets of iron and scintillating plastic. Some flexibility was built into the simulation allowing for variations in the sheet thicknesses and the detector cross section. The detector digitization was applied to the simulation 

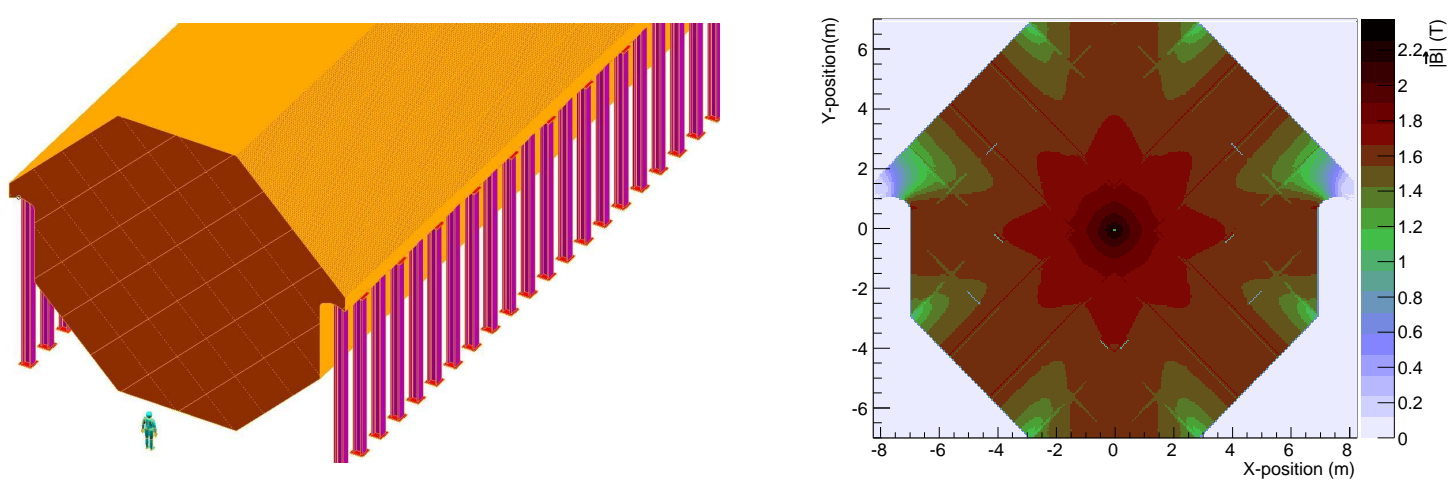

Figure 1: Left: schematic of a MIND for the neutrino factory. Right: the magnetic field expected in the steel plates

with a separate program to introduce signal attenuation and smearing. The digitized simulation was then passed to a reconstruction program.

Engineering feasibility studies have been completed in association with IDS-NF for a detector with an octagonal cross section 14 metres in height and width with steel plates $3 \mathrm{~cm}$ in thickness. Space point measurements in 3 dimensions are provided by scintillating planes consisting of two orthogonal arrays of bars $1 \mathrm{~cm}$ thick and $3.5 \mathrm{~cm}$ in width, so the total width of each detector module is $5 \mathrm{~cm}$ thick. A total detector mass of $100 \mathrm{kTonne}$ has been anticipated for a total length of $140 \mathrm{~m}$. This detector design has been reproduced in the simulation [9]. A graphical representation of this detector appears in Fig. 1 (left).

The magnetic field is provided by multiple turns of a superconducting transmission line (STL) providing a current of $100 \mathrm{kA} \cdot$ turn. A finite element simulation of the magnetic field in the steel has been completed and the results, as shown in Fig. 1 (right), has been used to provide field maps for the simulation and the event reconstruction. These field maps present a toroidal geometry which can be used to either focus positive or negative charged particles depending on the polarity of the current in the STL.

The reconstruction software was optimized for the identification of muons in neutrino charge current interactions. As a first step segments of single hit planes were identified by the pattern recognition algorithm. Additional hits were filtered into the trajectory using a Kalman algorithm provided by the RecPack toolkit [10]. This process was repeated to identify additional tracks, increasing the likelihood of reconstructing the muon track in the case where the muon track does not intersect the largest number of planes, as is the case when the muon appears at a high angle. The momentum of the trajectory is determined from the curvature of a helix fit to the trajectory using a Kalman algorithm. All charged particles that pass through more than five detector modules can be reconstructed with this algorithm necessitating the use of an event selection algorithm.

\section{Event Selection}

A multivariate analysis was adopted to further separate signal species from the backgrounds. The leading contributions to the background were primarily composed of pion punch through from 
neutral current and like-sign charge current neutrino interactions. Several methods, such as boosted decision trees[11] or the k-Nearest Neighbour (KNN) method, provided by the Toolkit for MultiVariate Analysis (TMVA)[12] released as part of the ROOT software package[13], have been evaluated for use with the wrong sign neutrino analysis assuming both muon charges. The selection used a set of six variables including the relative uncertainty in the measured trajectory curvature, the number of hits in a trajectory, and the mean energy along a trajectory among others. To select signal events the analysis was trained using a pure sample of charge current (CC) events as signal with a sample of neutral current (NC) events as a background. The analysis was then applied to independent simulations of $\left(\bar{v}_{\mu}, \bar{v}_{e}\right.$, and $\bar{v}_{\tau} \mathrm{CC}$ and NC events to establish the detector response for realistic neutrino events.

The detector efficiency after selection is shown in Fig. 2(left) assuming a detector that focuses positive charged particles using a KNN method. The accompanying backgrounds for stored $\mu^{-}$is shown in Fig. 2(right). The $1 \% \bar{v}_{\tau} \mathrm{CC}$ contamination serves as an additional signal channel for $\mathrm{CP}$ violation measurements.
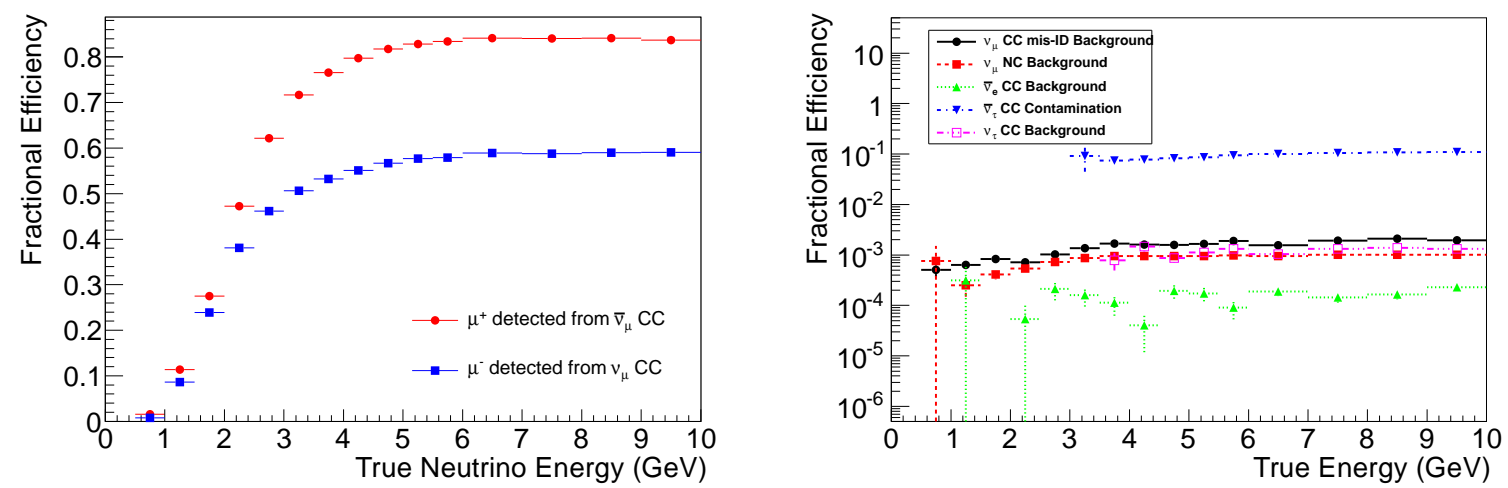

Figure 2: Event selection efficiencies from signal (left) and background (right) using a KNN method. Signals are shown for both stored muon charges while the background is shown for a stored $\mu^{-}$experiment.

\section{Physics at Neutrino Factories with MIND}

To establish an experimental sensitivity the detector response determined with the event selection was input into the GloBeS software package[14]. GloBeS predicted the interaction rates given a set of neutrino oscillation parameters after including matter effects between the far detector and the neutrino source. The data from the detector simulation were input into the GloBeS simulation in the form of matrices that map the predicted rates and resolution as a function of the true neutrino energy to the reconstructed rate as a function of the reconstructed neutrino energy.

The precision of the measurements of a $\mathrm{CP}$ violating phase, $\delta_{C P}$ was determined from the $\chi^{2}$ calculated from the rates with a given value of $\delta_{C P}$ and the case when $\delta_{C P}=0$. The sum of the two muon appearance channels $\left(\bar{v}_{e} \rightarrow \bar{v}_{\mu}\right)$ and the two muon disappearance channels were considered. Assuming a $100 \mathrm{kTon}$ detector $2000 \mathrm{~km}$ from a neutrino factory which stores $5 \times 10^{20}$ muons (of both charges) per year with a muon energy of $10 \mathrm{GeV}$, an experiment can achieve a precision of $4^{\circ}$ to $5^{\circ}$, or an $85 \%$ coverage of potential values of $\delta_{C P}$ at $3 \sigma$ after 10 years of data collection. The 

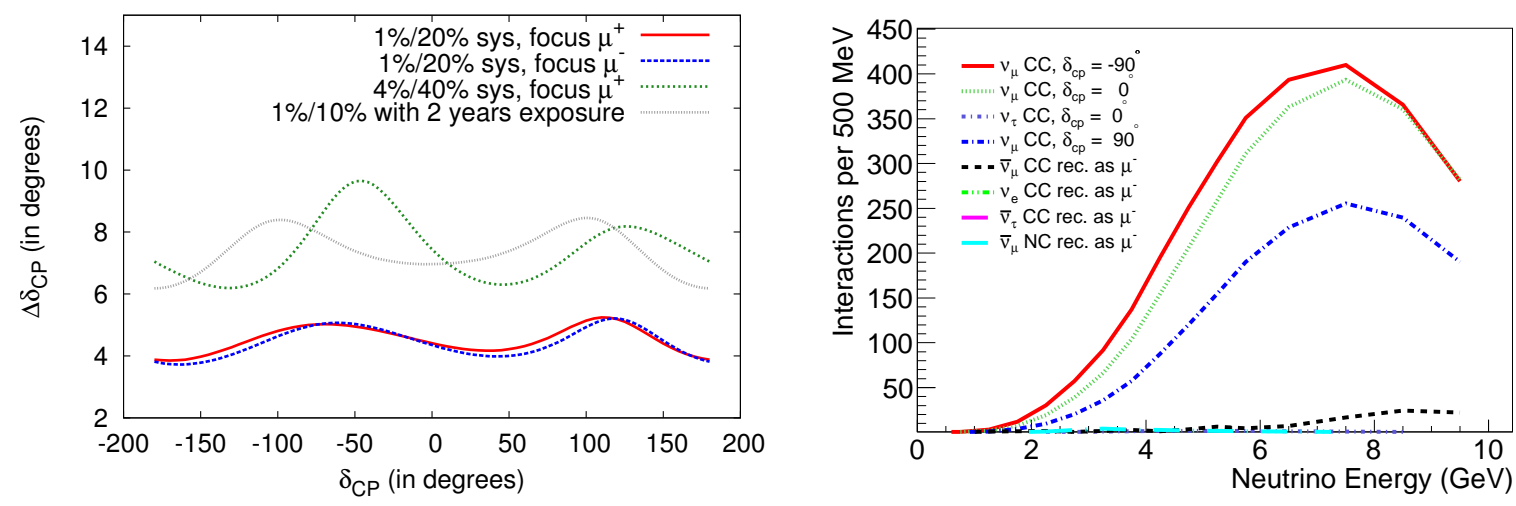

Figure 3: Precision of the CP violating phase $\delta_{C P}$ measured at a neutrino factory. For the IDS-NF baseline the precision as a function of $\delta_{C P}$ after a total exposure consisting of $5 \times 10^{21}$ muon decays of both charges delivered over 10 years is shown on the left with increased systematics and reduced exposure scenarios. The contributing event rates leading to this precision is shown on the right.

precision is shown in Fig. 3 (left) while the expected event rate is shown in Fig. 3 (right). The result did not significantly depend on the polarity of the detector magnetic field nor the selection method used.

The precision curves assumed a total systematic uncertainty of $1.4 \%$ for the signal and $20 \%$ for the background affecting the normalization alone. These uncertainties assumed that the source luminosity will be known at the $0.1 \%$ level and that measurements of the interaction cross-sections can be made at a neutrino factory facility at the $0.5 \%$ level. If the cross-sections can not be reevaluated, then this uncertainty dominates and the total uncertainty increases to $4 \%$ for the signal and $40 \%$ for the background. The precision in this case is between $6^{\circ}$ and $10^{\circ}$ which is roughly the equivalent of reducing exposure of the experiment by a factor of 5 . A breakdown of the sources of systematic error is given Table 2 .

\begin{tabular}{rcccc}
\hline Uncertainty & \multicolumn{2}{c}{ Known Measures } & \multicolumn{2}{c}{ Expected Contribution } \\
& Signal & Background & Signal & Background \\
\hline Source luminosity & $0.1 \%$ & $0.1 \%$ & $0.1 \%$ & $0.1 \%$ \\
Cross section & $4 \%$ & $40 \%$ & $0.5 \%$ & $5 \%$ \\
Hadronic Model & 0 & $15 \%$ & 0 & $8 \%$ \\
\hline
\end{tabular}

Table 2: The expected systematic uncertainties at a neutrino factory contrasted with the current state of the same systematic uncertainties assuming no input from the facility. A neutrino factory near detector program is expected to significantly reduce cross-sectional uncertainties as a consequence of the improved understanding of the neutrino flux.

The significance of cosmic ray backgrounds have also been explored for a neutrino factory. A simulation of cosmic rays in the neutrino factory MIND was produced using the CRY cosmic ray shower library [15] to evaluate the reconstruction efficiency of realistic cosmic muons. Given the expected neutrino factory duty factor, the exposed surface area of the detector volume, and a self veto cut removing trajectories that start in the outermost $30 \mathrm{~cm}$ of the detector volume an expected $2.6 \times 10^{5}$ cosmic muons contaminate a $v_{e} \rightarrow v_{\mu}$ appearance measurement and $7.3 \times 10^{5}$ cosmic 

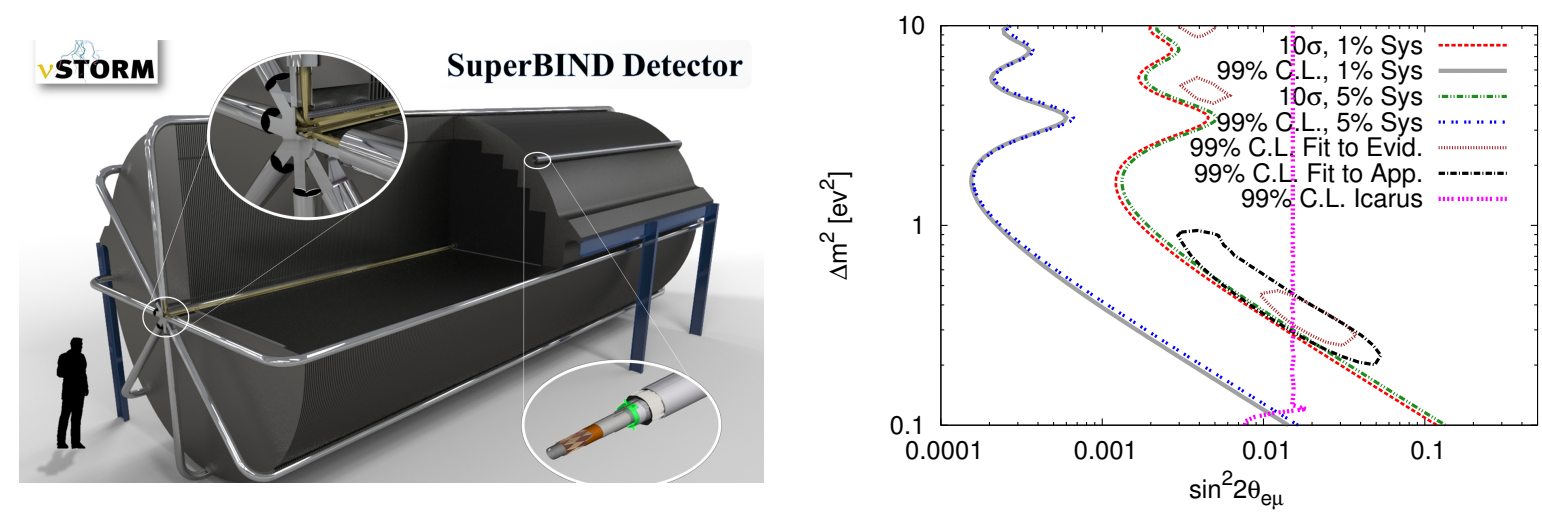

Figure 4: The proposed MIND detector to be used as the far detector in the nuSTORM short baseline oscillation measurements (left), known as SuperBIND. A short baseline $v_{\mu}$ appearance experiment using SuperBIND can make measurements with $10 \sigma$ significance within the region allowed by current global fits to the LSND, MiniBooNE, reactor, and gallium anomaly data (Evid. Data) or the global fits of all available neutrino appearance data (App. Data) (right)[16].

events contaminating a $v_{e} \rightarrow v_{\mu}$ appearance measurement after 10 years of exposure. To reduce $2.6 \times 10^{5}$ events to less than $10 \%$ of the corresponding background events (298) approximately $6 \mathrm{~m}$ of rock overburden is required.

\subsection{Reducing the Neutrino Factory Baseline}

Alternative neutrino factory baselines have also been considered. For a neutrino factory to operate within a baseline of $1300 \mathrm{~km}$, consistent with the baseline between FermiLab and SURF, the optimal beam energy for $\mathrm{CP}$ violation studies is $5 \mathrm{GeV}$. The consequence of this decreased energy is that the peak of the oscillated spectrum is in the rising region of the $\mathrm{CC}$ interaction detection efficiency curve, which results in a precision in $\delta_{C P}$ between $12^{\circ}$ and $23^{\circ}$ for a $100 \mathrm{kTon}$ MIND exposed to $5 \times 10^{20}$ muons of both species per year over 10 years. This $\delta_{C P}$ precision is comparable to the sensitivity of conventional long-baseline facilities. This may be improved if the platinum channel, $\bar{v}_{\mu} \rightarrow \bar{v}_{\mu}$, can also be exploited. Therefore it has been concluded that a different detector from the IDS-NF MIND is required for high precision CP violation studies at a $5 \mathrm{GeV}$ neutrino factory.

\subsection{A Near Term Neutrino Factory: nuSTORM}

A third neutrino factory facility, called Neutrinos from Stored Muons (nuSTORM) has been proposed which may be constructed entirely with existing technology[17, 18]. nuSTORM transports pions from a conventional proton target in a magnetic horn assembly through a chicane into a muon storage ring without acceleration or cooling. The proposed storage ring then retains muons within a momentum acceptance of $3.8 \mathrm{GeV} \pm 10 \%$ that result from the pions that decay within the first straight. Unlike the other neutrino factories, he neutrinos that result from the pion decays may also be used making nuSTORM a three flavour neutrino source. For example, if the facility accepts positive particles, then nuSTORM produces $v_{\mu}, \bar{v}_{\mu}$, and $v_{e}$ beams, with the $v_{\mu}$ beam appearing immediately after injection and all three with extremely low backgrounds. A 1.3 kTon variation 
of MIND (called SuperBIND) has been developed to measure short baseline oscillations at nuSTORM with a cylindrical geometry $3 \mathrm{~m}$ in diameter and $13 \mathrm{~m}$ in length. This MIND possesses a large magnetic field, induced by $270 \mathrm{kA} \cdot$ turns carried by multiple turns of STL, $1.5 \mathrm{~cm}$ thick steel plates, and scintillator planes composed of paired arrays of scintillator bars in the $\mathrm{x}$ and $\mathrm{y}$ directions, $0.75 \mathrm{~cm}$ thick each. A short baseline experiment using SuperBIND can definitively evaluate the presence of $\mathrm{eV}$ scale sterile neutrinos.

Using the same simulation, reconstruction, and analysis as the long baseline experiment, it has been demonstrated that a MIND at $2 \mathrm{~km}$ away from nuSTORM can make a $10 \sigma$ measurement of a sterile neutrino consistent with the current global fits to the LSND and MiniBooNE experiments[16] using the $v_{\mu}$ appearance channel alone. A highly restrictive event selection which reduces the backgrounds to $10^{-5}$ of the initial number of contributing neutrino interactions made the signal significance of $10 \sigma$ possible[19]. Other channels may also be evaluated with nuSTORM including the $v_{\mu}$ and $\bar{v}_{\mu}$ disappearance channels. There is also potential to evaluate the $v_{e}$ appearance channel within the same time scale as the $v_{\mu}$ appearance measurement using SuperBIND. Simulation has shown that a $v_{e}$ detection efficiency on the order of $10 \%$ is possible with $\mathrm{Su}-$ perBIND. Because the pion source at nuSTORM provides a flux that is more than 10 times that of the flux from the muon decays this efficiency can make a significant measurement in the region preferred by the existing evidence.

\section{Conclusions}

Magnetized iron neutrino detectors are a well developed technology suitable for a neutrino factory. To evaluate the sensitivity of future neutrino factory facilities for both short baseline and long baseline experiments a simulation of a MIND has been developed. With a complete muon track reconstruction algorithm and a charge current event selection algorithm, a reduction of backgrounds for wrong-sign neutrino oscillation measurements below parts in $10^{3}$ have been demonstrated allowing a measured precision in the measurement of $\delta_{C P}$ between $4^{\circ}$ and $5^{\circ}$ is achievable at the IDS-NF model of the neutrino factory. Other potential neutrino facilities have been evaluated with this simulation, including the short baseline oscillation searches at nuSTORM, and CP violation at a lowered energy neutrino factory (NuMAX). The MIND simulation is also being applied to studies in other contexts not under discussion including of a MIND proto-type under construction by the AiDA collaboration at CERN for the purpose of validation of the methods used here[20], and interaction studies of a near detector at nuSTORM.

\section{References}

[1] P. Adamson et al., "The MINOS calibration detector," Nucl. Instrum. Meth., vol. A556, pp. 119-133, 2006.

[2] P. Adamson et al., "Neutrino and Antineutrino Inclusive Charged-current Cross Section Measurements with the MINOS Near Detector," Phys.Rev., vol. D81, p. 072002, 2010.

[3] P. Adamson et al., "New constraints on muon-neutrino to electron-neutrino transitions in MINOS," Phys. Rev. D, vol. 82, p. 051102, 2010. 
[4] S. Choubey et al., "International Design Study for the Neutrino Factory, Interim Design Report," 2011.

[5] F. An et al., "Observation of electron-antineutrino disappearance at Daya Bay," Phys.Rev.Lett., vol. 108, p. 171803, 2012.

[6] R. Bayes, A. Laing, F. J. P. Soler, A. Cervera Villanueva, J. J. Gomez Cadenas, et al., "The Golden Channel at a Neutrino Factory revisited: improved sensitivities from a Magnetised Iron Neutrino Detector," Phys.Rev., vol. D86, p. 093015, 2012.

[7] C. Andreopoulos et al., "The GENIE Neutrino Monte Carlo Generator," Nucl. Instrum. Meth. A, vol. 614, pp. 87-104, 2010.

[8] J. Apostolakis and D. H. Wright, “An overview of the GEANT4 toolkit," AIP Conf. Proc., vol. 896, pp. 1-10, 2007.

[9] A. Bross, R. Wands, R. Bayes, A. Laing, F. Soler, et al., "Toroidal magnetized iron neutrino detector for a neutrino factory," Phys.Rev.ST Accel.Beams, vol. 16, no. 8, p. 081002, 2013.

[10] A. Cervera-Villanueva, J. J. Gomez-Cadenas, and J. A. Hernando, “'RecPack' a reconstruction toolkit," Nucl. Instrum. Meth., vol. A534, pp. 180-183, 2004.

[11] H.-J. Yang, T. Dai, A. Wilson, Z. Zhao, and B. Zhou, "A multivariate training technique with event reweighting," Journal of Instrumentation, vol. 3, 082007.

[12] A. Hoecker, J. Stelzer, F. Tegenfeldt, H. Voss, K. Voss, et al., "TMVA - Toolkit for Multivariate Data Analysis," PoS, vol. ACAT, p. 040, 2007.

[13] R. Brun and F. Rademakers, "ROOT: An object oriented data analysis framework," Nucl. Instrum. Meth., vol. A389, pp. 81-86, 1997.

[14] P. Huber, M. Lindner, and W. Winter, "Simulation of long-baseline neutrino oscillation experiments with GLoBES,” Comput. Phys. Commun., vol. 167, p. 195, 2005.

[15] C. Hagmann, D. Lange, J. Verbeke, and D. Wright, “Cosmic-ray shower library (cry),” Tech. Rep. UCRL-TM-229453, Lawrence Livermore National Laboratory, 2013.

[16] J. Kopp, P. A. N. Machado, M. Maltoni, and T. Schwetz, "Sterile Neutrino Oscillations: The Global Picture," JHEP, vol. 1305, p. 050, 2013.

[17] D. Adey, S. Agarwalla, C. Ankenbrandt, R. Asfandiyarov, J. Back, et al., "Neutrinos from Stored Muons nuSTORM: Expression of Interest,” 2013.

[18] D. Adey et al., "nuSTORM - Neutrinos from STORed Muons: Proposal to the Fermilab PAC," 2013.

[19] D. Adey et al., "Light sterile neutrino sensitivity at the nuSTORM facility," Phys.Rev., vol. D89, p. $071301,2014$.

[20] R. Asfandiyarov, R. Bayes, A. Blondel, M. Bogomilov, A. Bross, et al., "Proposal for SPS beam time for the baby MIND and TASD neutrino detector prototypes," 2014. 